\title{
The Barut Second-Order Equation: Lagrangian, Dynamical Invariants and Interactions
}

\author{
Valeri V. Dvoeglazov
}

\begin{abstract}
The second-order equation in the $(1 / 2,0) \oplus(0,1 / 2)$ representation of the Lorentz group has been proposed by A. Barut in the 70s, ref. [1]. It permits to explain the mass splitting of leptons $(e, \mu, \tau)$. Recently, the interest has grown to this model (see, for instance, the papers by S. Kruglov 2 and J. P. Vigier et al. [3]). We continue the research deriving the equation from the first principles, finding dynamical invariants for this model, investigating the influence of potential interactions.
\end{abstract}

\section{Introduction}

The Barut main equation is

$$
\left[i \gamma_{\mu} \partial_{\mu}-\alpha_{2} \frac{\partial_{\mu} \partial_{\mu}}{m}+\kappa\right] \Psi=0 .
$$

- It represents a theory with the conserved current that is linear in 15 generators of the 4-dimensional representation of the $O(4,2)$ group, $N_{a b}=\frac{i}{2} \gamma_{a} \gamma_{b}, \gamma_{a}=\left\{\gamma_{\mu}, \gamma_{5}, i\right\}$.

- Instead of 4 solutions it has 8 solutions with the correct relativistic relation $E= \pm \sqrt{\mathbf{p}^{2}+m_{i}^{2}}$. In fact, it describes states of different masses (the second one is $m_{\mu}=m_{e}\left(1+\frac{3}{2 \alpha}\right), \alpha$ is the fine structure constant), provided that a certain physical condition is imposed on the $\alpha_{2}$ parameter (the anomalous magentic moment should be equal to $4 \alpha / 3)$.

- One can also generalize the formalism to include the third state, the $\tau$ - lepton [1b].

- Barut has indicated at the possibility of including $\gamma_{5}$ terms ( e.g., $\left.\sim \gamma_{5} \kappa^{\prime}\right)$. 


\section{Main Results}

If we present the 4-spinor as $\left.\Psi(\mathbf{p})=\operatorname{column}\left(\phi_{R}(\mathbf{p})\right) \quad \phi_{L}(\mathbf{p})\right)$ then Ryder states [5] that $\phi_{R}(\mathbf{0})=\phi_{L}(\mathbf{0})$. Similar argument has been given by Faustov [6]: "the matrix $B$ exists such that $B u_{\lambda}(\mathbf{0})=u_{\lambda}(\mathbf{0}), B^{2}=I$ for any $(2 J+1)$ - component function within the Lorentz invariant theories". The latter statement is more general than the Ryder one, because it admits

$$
B=\left(\begin{array}{cc}
0 & e^{+i \alpha} \\
e^{-i \alpha} & 0
\end{array}\right)
$$

so that $\phi_{R}(\mathbf{0})=e^{i \alpha} \phi_{L}(\mathbf{0})$. The most general form of the relation in the $(1 / 2,0) \oplus(0,1 / 2)$ representation has been given by Dvoeglazov [7,4a]:

$$
\phi_{L}^{h}(\mathbf{0})=a(-1)^{\frac{1}{2}-h} e^{i\left(\theta_{1}+\theta_{2}\right)} \Theta_{1 / 2}\left[\phi_{L}^{-h}(\mathbf{0})\right]^{*}+b e^{2 i \theta_{h}} \Xi_{1 / 2}^{-1}\left[\phi_{L}^{h}(\mathbf{0})\right]^{*},
$$

with

$$
\Theta_{1 / 2}=\left(\begin{array}{cc}
0 & -1 \\
1 & 0
\end{array}\right)=-i \sigma_{2}, \quad \Xi_{1 / 2}=\left(\begin{array}{cc}
e^{i \varphi} & 0 \\
0 & e^{-i \varphi}
\end{array}\right)
$$

$\Theta_{J}$ is the Wigner operator for spin $J=1 / 2, \varphi$ is the azimuthal angle $\mathbf{p} \rightarrow 0$ of the spherical coordinate system.

Next, we use the Lorentz transformations:

$$
\Lambda_{R, L}=\exp ( \pm \sigma \cdot \phi / 2), \cosh \phi=E_{p} / m, \sinh \phi=|\mathbf{p}| / m, \hat{\phi}=\mathbf{p} /|\mathbf{p}| .
$$

Applying the boosts and the relations between spinors in the rest frame, one can obtain:

$$
\begin{aligned}
& \phi_{L}^{h}(\mathbf{p})=a \frac{\left(p_{0}-\sigma \cdot \mathbf{p}\right)}{m} \phi_{R}^{h}(\mathbf{p})+b(-1)^{1 / 2+h} \Theta_{1 / 2} \Xi_{1 / 2} \phi_{R}^{-h}(\mathbf{p}), \\
& \phi_{R}^{h}(\mathbf{p})=a \frac{\left(p_{0}+\sigma \cdot \mathbf{p}\right)}{m} \phi_{L}^{h}(\mathbf{p})+b(-1)^{1 / 2+h} \Theta_{1 / 2} \Xi_{1 / 2} \phi_{L}^{-h}(\mathbf{p}) .
\end{aligned}
$$

$\left(\theta_{1}=\theta_{2}=0, p_{0}=E_{p}=\sqrt{\mathbf{p}^{2}+m^{2}}\right)$. In the Dirac form we have:

$$
\left[a \frac{\hat{p}}{m}-1\right] u_{h}(\mathbf{p})+i b(-1)^{\frac{1}{2}-h} \gamma^{5} \mathcal{C} u_{-h}^{*}(\mathbf{p})=0,
$$

where $\mathcal{C}=\left(\begin{array}{cc}0 & i \Theta_{1 / 2} \\ -i \Theta_{1 / 2} & 0\end{array}\right)$, the charge conjugate operator. In the QFT form we must introduce the creation/annihilation operators. Let $b_{\downarrow}=$ $-i a_{\uparrow}, b_{\uparrow}=+i a_{\downarrow}$, then

$$
\left[a \frac{i \gamma^{\mu} \partial_{\mu}}{m}+b \mathcal{C K}-1\right] \Psi\left(x^{\nu}\right)=0 .
$$


If one applies the unitary transformation to the Majorana representation [8]

$$
\mathcal{U}=\frac{1}{2}\left(\begin{array}{cc}
1-i \Theta_{1 / 2} & 1+i \Theta_{1 / 2} \\
-1-i \Theta_{1 / 2} & 1-i \Theta_{1 / 2}
\end{array}\right), \mathcal{U C K U}^{-1}=-\mathcal{K}
$$

then $\gamma$-matrices become to be pure imaginary, and the equations are pure real.

$$
\begin{aligned}
& {\left[a \frac{i \hat{\partial}}{m}-b-1\right] \Psi_{1}=0,} \\
& {\left[a \frac{i \hat{\partial}}{m}+b-1\right] \Psi_{2}=0,}
\end{aligned}
$$

where $\Psi=\Psi_{1}+i \Psi_{2}$. It appears as if the real and imaginary parts of the field have different masses. Finally, for superpositions $\phi=\Psi_{1}+\Psi_{2}$, $\chi=\Psi_{1}-\Psi_{2}$, multiplying by $b \neq 0$ we have:

$$
\left[2 a \frac{i \gamma^{\mu} \partial_{\mu}}{m}+a^{2} \frac{\partial^{\mu} \partial_{\mu}}{m^{2}}+b^{2}-1\right] \frac{\phi\left(x^{\nu}\right)}{\chi\left(x^{\nu}\right)}=0,
$$

If we put $a / 2 m \rightarrow \alpha_{2}, \frac{1-b^{2}}{2 a} m \rightarrow \kappa$ we recover the Barut equation.

How can we get the third lepton state? See the refs. [1b,4b]:

$$
M_{\tau}=M_{\mu}+\frac{3}{2} \alpha^{-1} n^{4} M_{e}=M_{e}+\frac{3}{2} \alpha^{-1} 1^{4} M_{e}+\frac{3}{2} \alpha^{-1} 2^{4} M_{e}=1786.08 \mathrm{MeV} .
$$

The physical origin was claimed by Barut to be in the magnetic selfinteraction of the electron (the factor $n^{4}$ appears due to the Bohr-Sommerfeld rule for the charge moving in circular orbits in the field of a fixed magnetic dipole $\mu$ ). One can start from (2.6), but, as opposed to the abovementioned consideration, one can write the coordinate-space equation in the form:

$$
\left[a \frac{i \gamma^{\mu} \partial_{\mu}}{m}+b_{1} \mathcal{C K}-1\right] \Psi\left(x^{\nu}\right)+b_{2} \gamma^{5} \mathcal{C K} \tilde{\Psi}\left(x^{\nu}\right)=0,
$$

with $\Psi^{M R}=\Psi_{1}+i \Psi_{2}, \tilde{\Psi}^{M R}=\Psi_{3}+i \Psi_{4}$. As a result,

$$
\begin{aligned}
& \left(a \frac{i \gamma^{\mu} \partial_{\mu}}{m}-1\right) \phi-b_{1} \chi+i b_{2} \gamma^{5} \tilde{\phi}=0, \\
& \left(a \frac{i \gamma^{\mu} \partial_{\mu}}{m}-1\right) \chi-b_{1} \phi-i b_{2} \gamma^{5} \tilde{\chi}=0 .
\end{aligned}
$$

The operator $\tilde{\Psi}$ may be linear-dependent on the states included in the $\Psi$. let us apply the most simple form $\Psi_{1}=-i \gamma^{5} \Psi_{4}, \Psi_{2}=+i \gamma^{5} \Psi_{3}$. Then, one can recover the $3 r d$ order Barut-like equation [4b]:

$$
\left[i \gamma^{\mu} \partial_{\mu}-m \frac{1 \pm b_{1} \pm b_{2}}{a}\right]\left[i \gamma^{\nu} \partial_{\nu}+\frac{a}{2 m} \partial^{\nu} \partial_{\nu}+m \frac{b_{1}^{2}-1}{2 a}\right] \Psi_{1,2}=0 .
$$


It is simply the product of 3 Dirac equations with different masses. Thus, we have three mass states.

Let us reveal the connections with other models. For instance, in refs. 3 , 9. the following equation has been studied:

$$
\begin{aligned}
& {\left[(i \hat{\partial}-e \hat{A})(i \hat{\partial}-e \hat{A})-m^{2}\right] \Psi=} \\
& \quad=\left[\left(i \partial_{\mu}-e A_{\mu}\right)\left(i \partial^{\mu}-e A^{\mu}\right)-\frac{1}{2} e \sigma^{\mu \nu} F_{\mu \nu}-m^{2}\right] \Psi=0
\end{aligned}
$$

for the 4-component spinor $\Psi$. This is the Feynman-Gell-Mann equation. In the free case we have the Lagrangian (see Eq. (9) of ref. [3c]):

$$
\mathcal{L}_{0}=(i \overline{\hat{\partial} \Psi})(i \hat{\partial} \Psi)-m^{2} \bar{\Psi} \Psi
$$

We can note:

- The Barut equation is the sum of the Dirac equation and the FeynmanGell-Mann equation.

- Recently, it was suggested to associate an analogue of Eq. (2.18) with the dark matter [10, provided that $\Psi$ is composed of the self/antiself charge conjugate spinors, and it has the dimension [energy $]^{1}$ in $c=\hbar=1$. The interaction Lagrangian is $\mathcal{L}^{H} \sim g \bar{\Psi} \Psi \phi^{2}$.

- The term $\sim \bar{\Psi} \sigma^{\mu \nu} \Psi F_{\mu \nu}$ will affect the photon propagation, and nonlocal terms will appear in higher orders.

- However, it was shown in [3b,c] that a) the Mott cross-section formula (which represents the Coulomb scattering up to the order $\sim e^{2}$ ) is still valid; b) the hydrogen spectrum is not much disturbed; if the electromagnetic field is weak the corrections are small.

- The solutions are the eigenstates of $\gamma^{5}$ operator.

- In general, $J_{0}$ is not the positive-defined quantity, since the general solution $\Psi=a \Psi_{+}+b \Psi_{-}$, where $\left[i \gamma^{\mu} \partial_{\mu} \pm m\right] \Psi_{ \pm}=0$, see also [11].

The most general conserved current of the Barut-like theories is

$$
J_{\mu}=\alpha_{1} \gamma_{\mu}+\alpha_{2} p_{\mu}+\alpha_{3} \sigma_{\mu \nu} q^{\nu} .
$$

Let us try the Lagrangian:

$$
\begin{gathered}
\mathcal{L}=\mathcal{L}_{\text {Dirac }}+\mathcal{L}_{\text {add }}, \\
\mathcal{L}_{\text {Dirac }}=\alpha_{1}\left[\bar{\Psi} \gamma^{\mu}\left(\partial_{\mu} \Psi\right)-\left(\partial_{\mu} \bar{\Psi}\right) \gamma^{\mu} \Psi\right]-\alpha_{4} \bar{\Psi} \Psi \\
\mathcal{L}_{\text {add }}=\alpha_{2}\left(\partial_{\mu} \bar{\Psi}\right)\left(\partial^{\mu} \Psi\right)+\alpha_{3} \partial_{\mu} \bar{\Psi} \sigma^{\mu \nu} \partial_{\nu} \Psi
\end{gathered}
$$


Then, the equation follows:

$$
\left[2 \alpha_{1} \gamma^{\mu} \partial_{\mu}-\alpha_{2} \partial_{\mu} \partial^{\mu}-\alpha_{4}\right] \Psi=0
$$

and its Dirac-conjugate:

$$
\bar{\Psi}\left[2 \alpha_{1} \gamma^{\mu} \partial_{\mu}+\alpha_{2} \partial_{\mu} \partial^{\mu}+\alpha_{4}\right]=0 .
$$

The derivatives acts to the left in the second equation. Thus, we have the Dirac equation when $\alpha_{1}=\frac{i}{2}, \alpha_{2}=0$, and the Barut equation when $\alpha_{2}=\frac{1}{m} \frac{2 \alpha / 3}{1+4 \alpha / 3}$.

In the Euclidean metrics the dynamical invariants are

$$
\begin{gathered}
\mathcal{J}_{\mu}=-i \sum_{i}\left[\frac{\partial \mathcal{L}}{\partial\left(\partial_{\mu} \Psi_{i}\right)} \Psi_{i}-\bar{\Psi}_{i} \frac{\partial \mathcal{L}}{\partial\left(\partial_{\mu} \bar{\Psi}_{i}\right)}\right] \\
\mathcal{T}_{\mu \nu}=-\sum_{i}\left[\frac{\partial \mathcal{L}}{\partial\left(\partial_{\mu} \Psi_{i}\right)} \partial_{\nu} \Psi_{i}+\partial_{\nu} \bar{\Psi}_{i} \frac{\partial \mathcal{L}}{\partial\left(\partial_{\mu} \bar{\Psi}_{i}\right)}\right]+\mathcal{L} \delta_{\mu \nu} \\
\mathcal{S}_{\mu \nu, \lambda}=-i \sum_{i j}\left[\frac{\partial \mathcal{L}}{\partial\left(\partial_{\lambda} \Psi_{i}\right)} N_{\mu \nu, i j}^{\Psi} \Psi_{j}+\bar{\Psi}_{i} N_{\mu \nu, i j}^{\bar{\Psi}} \frac{\partial \mathcal{L}}{\partial\left(\partial_{\lambda} \bar{\Psi}_{j}\right)}\right]
\end{gathered}
$$

$N_{\mu \nu}^{\Psi, \bar{\Psi}}$ are the Lorentz group generators.

Then, the energy-momentum tensor is

$$
\begin{aligned}
& \mathcal{T}_{\mu \nu}=-\alpha_{1}\left[\bar{\Psi} \gamma_{\mu} \partial_{\nu} \Psi-\partial_{\nu} \bar{\Psi} \gamma_{\mu} \Psi\right]-\alpha_{2}\left[\partial_{\mu} \bar{\Psi} \partial_{\nu} \Psi+\partial_{\nu} \bar{\Psi} \partial_{\mu} \Psi\right]- \\
& \quad-\alpha_{3}\left[\partial_{\alpha} \bar{\Psi} \sigma_{\alpha \mu} \partial_{\nu} \Psi++\partial_{\nu} \bar{\Psi} \sigma_{\mu \alpha} \partial_{\alpha} \Psi\right]+\left[\alpha_{1}\left(\bar{\Psi} \gamma_{\mu} \partial_{\mu} \Psi-\partial_{\mu} \bar{\Psi} \gamma_{\mu} \Psi\right)+\right. \\
& \left.\quad+\alpha_{2} \partial_{\alpha} \bar{\Psi} \partial_{\alpha} \Psi+\alpha_{3} \partial_{\alpha} \bar{\Psi} \sigma_{\alpha \beta} \partial_{\beta} \Psi+\alpha_{4} \bar{\Psi} \Psi\right] \delta_{\mu \nu}
\end{aligned}
$$

Hence, the Hamiltonian $\hat{\mathcal{H}}=-i P_{4}=-\int \mathcal{T}_{44} d^{3} x$ is

$$
\begin{aligned}
\hat{\mathcal{H}} & =\int d^{3} x\left\{\alpha_{1}\left[\partial_{i} \bar{\Psi} \gamma_{i} \Psi-\bar{\Psi} \gamma_{i} \partial_{i} \Psi\right]+\alpha_{2}\left[\partial_{4} \bar{\Psi} \partial_{4} \Psi-\partial_{i} \bar{\Psi} \partial_{i} \Psi\right]-\right. \\
& \left.-\alpha_{3}\left[\partial_{i} \bar{\Psi} \sigma_{i j} \partial_{j} \Psi\right]-\alpha_{4} \bar{\Psi} \Psi\right\} .
\end{aligned}
$$

The 4-current is

$$
\mathcal{J}_{\mu}=-i\left\{2 \alpha_{1} \bar{\Psi} \gamma_{\mu} \Psi+\alpha_{2}\left[\left(\partial_{\mu} \bar{\Psi}\right) \Psi-\bar{\Psi}\left(\partial_{\mu} \Psi\right)\right]+\alpha_{3}\left[\partial_{\alpha} \bar{\Psi} \sigma_{\alpha \mu} \Psi-\bar{\Psi} \sigma_{\mu \alpha} \partial_{\alpha} \Psi\right]\right\} .
$$

Hence, the charge operator $\hat{\mathcal{Q}}=-i \int \mathcal{J}_{4} d^{3} x$ is

$$
\hat{\mathcal{Q}}=-\int\left\{2 \alpha_{1} \Psi^{\dagger} \Psi+\alpha_{2}\left[\left(\partial_{4} \bar{\Psi}\right) \Psi-\bar{\Psi}\left(\partial_{4} \Psi\right)\right]+\alpha_{3}\left[\partial_{i} \bar{\Psi} \sigma_{i 4} \Psi-\bar{\Psi} \sigma_{4 i} \partial_{i} \Psi\right]\right\} d^{3} x
$$

Finally, the spin tensor is

$$
\begin{aligned}
\mathcal{S}_{\mu \nu, \lambda}= & -\frac{i}{2}\left\{\alpha_{1}\left[\bar{\Psi} \gamma_{\lambda} \sigma_{\mu \nu} \Psi+\bar{\Psi} \sigma_{\mu \nu} \gamma_{\lambda} \Psi\right]+\alpha_{2}\left[\partial_{\lambda} \bar{\Psi} \sigma_{\mu \nu} \Psi-\bar{\Psi} \sigma_{\mu \nu} \partial_{\lambda} \Psi\right]+\right. \\
& \left.+\alpha_{3}\left[\partial_{\alpha} \bar{\Psi} \sigma_{\alpha \lambda} \sigma_{\mu \nu} \Psi-\bar{\Psi} \sigma_{\mu \nu} \sigma_{\lambda \alpha} \partial_{\alpha} \Psi\right]\right\}
\end{aligned}
$$


In the quantum case the corresponding field operators are written:

$$
\begin{aligned}
\Psi\left(x^{\mu}\right) & =\sum_{h} \int \frac{d^{3} \mathbf{p}}{(2 \pi)^{3}}\left[u_{h}(\mathbf{p}) a_{h}(\mathbf{p}) e^{+i p \cdot x}+v_{h}(\mathbf{p}) b_{h}^{\dagger}(\mathbf{p}) e^{-i p \cdot x}\right],(2 \\
\bar{\Psi}\left(x^{\mu}\right) & =\sum_{h} \int \frac{d^{3} \mathbf{p}}{(2 \pi)^{3}}\left[\bar{u}_{h}(\mathbf{p}) a_{h}^{\dagger}(\mathbf{p}) e^{-i p \cdot x}+\bar{v}_{h}(\mathbf{p}) b_{h}(\mathbf{p}) e^{+i p \cdot x}\right] .(2 .
\end{aligned}
$$

The 4-spinor normalization is

$$
\bar{u}_{h} u_{h^{\prime}}=\delta_{h h^{\prime}}, \quad \bar{v}_{h} v_{h^{\prime}}=-\delta_{h h^{\prime}} .
$$

The commutation relations are

$$
\begin{aligned}
{\left[a_{h}(\mathbf{p}), a_{h^{\prime}}^{\dagger}(\mathbf{k})\right]_{+} } & =(2 \pi)^{3} \frac{m}{p_{4}} \delta^{(3)}(\mathbf{p}-\mathbf{k}) \delta_{h h^{\prime}}, \\
{\left[b_{h}(\mathbf{p}), b_{h^{\prime}}^{\dagger}(\mathbf{k})\right]_{+} } & =(2 \pi)^{3} \frac{m}{p_{4}} \delta^{(3)}(\mathbf{p}-\mathbf{k}) \delta_{h h^{\prime}},
\end{aligned}
$$

with all other being equal to zero. The dimensions of the $\Psi, \bar{\Psi}$ are as usual, [energy $]^{3 / 2}$. Hence, the second-quantized Hamiltonian is written

$$
\hat{\mathcal{H}}=-\sum_{h} \int \frac{d^{3} \mathbf{p}}{(2 \pi)^{3}} \frac{2 E_{p}^{2}}{m}\left[\alpha_{1}+m \alpha_{2}\right]:\left[a_{h}^{\dagger} a_{h}-b_{h} b_{h}^{\dagger}\right]: .
$$

(Remember that $\alpha_{1} \sim \frac{i}{2}$, the commutation relations may give another $i$, so the contribution of the first term to eigenvalues will be real. But if $\alpha_{2}$ is real, the contribution of the second term may be imaginary). The charge is

$\hat{\mathcal{Q}}=-\sum_{h h^{\prime}} \int \frac{d^{3} \mathbf{p}}{(2 \pi)^{3}} \frac{2 E_{p}}{m}\left[\left(\alpha_{1}+m \alpha_{2}\right) \delta_{h h^{\prime}}-i \alpha_{3} \bar{u}_{h} \sigma_{i 4} p_{i} u_{h^{\prime}}\right]:\left[a_{h}^{\dagger} a_{h^{\prime}}+b_{h} b_{h^{\prime}}^{\dagger}\right]:$.

However, due to $\left[\Lambda_{R, L}, \sigma \cdot \mathbf{p}\right]_{-}=0$ the last term with $\alpha_{3}$ does not contribute.

\section{Conclusions}

The conclusions are:

- We obtained the Barut-like equations of the 2nd order and 3rd order in derivatives. The Majorana representation has been used.

- We obtained dynamical invariants for the free Barut field on the classical and quantum level. 
- We found relations with other models (such as the Feynman-GellMann equation).

- As a result of analysis of dynamical invariants, we can state that at the free level the term $\sim \alpha_{3} \partial_{\mu} \bar{\Psi} \sigma_{\mu \nu} \partial_{\nu} \Psi$ in the Lagrangian does not contribute.

- However, the interaction terms $\sim \alpha_{3} \bar{\Psi} \sigma_{\mu \nu} \partial_{\nu} \Psi A_{\mu}$ will contribute when we construct the Feynman diagrams and the $S$-matrix. In the curved space (the 4-momentum Lobachevsky space) the influence of such terms has been investigated in the Skachkov works [12. Briefly, the contribution will be such as if the 4-potential were interact with some "renormalized" spin. Perhaps, this explains, why did Barut use the classical anomalous magnetic moment $g \sim 4 \alpha / 3$ instead of $\frac{\alpha}{2 \pi}$.

\section{Acknowledgements}

The author acknowledges discussions with participants of recent conferences. I am grateful to an anonymous referee for the reference [13], which develops the ideas of ref. [1], discussing renormalizability of the quantum

field theory in the presence of three (and more) families when the electromagnetic interactions are included.

\section{REFERENCES}

[1] A. O. Barut, Phys. Lett. B73, 310 (1978); Phys. Rev. Lett. 42, 1251 (1979); R. Wilson, Nucl. Phys. B68, 157 (1974).

[2] S. I. Kruglov, quant-ph/0408056 Ann. Fond. Broglie 29, No. H2 (the special issue dedicated to Yang and Mills, ed. by V. Dvoeglazov et al.).

[3] N. C. Petroni, J. P. Vigier et al, Nuovo Cim. B81, 243 (1984); Phys. Rev. D30, 495 (1984); ibid. D31, 3157 (1985).

[4] V. V. Dvoeglazov, Int. J. Theor. Phys. 37, 1909 (1998); Ann. Fond Broglie 25, 81 (2000).

[5] L. M. Ryder, Quantum Field Theory. (Cambridge University Press, 1985).

[6] R. N. Faustov, Preprint ITF-71-117P, Kiev, Sept. 1971.

[7] V. V. Dvoeglazov, Hadronic J. Suppl. 10, 349 (1995).

[8] V. V. Dvoeglazov, Int. J. Theor. Phys. 36, 635 (1997).

[9] R. Feynman and M. Gell-Mann, Phys. Rev. 109, 193 (1958).

[10] D. V. Ahluwalia and D. Grumiller, hep-th/0410192.

[11] M. Markov, ZhETF 7, 579; ibid., 603 (1937); Nucl. Phys. 55, 130 (1964).

[12] N. B. Skachkov, Theor. Math. Phys. 22, 149 (1975); ibid. 25, 1154 (1976).

[13] A. O. Barut and J. P. Crawford, Phys. Lett. B82, 233 (1979); J. P. Crawford and A. O. Barut, Phys. Rev. D27, 2493 (1983). 
Valeri V. Dvoeglazov

Universidad de Zacatecas

Apartado Postal 636, Suc. UAZ

Zacatecas, 98062 Zacatecas

E-mail: valeri@planck.reduaz.mx

Submitted: September 22, 2005; Revised: December 15, 2006. 